\title{
SOME INEQUALITIES FOR THE TRIGAMMA FUNCTION IN TERMS OF THE DIGAMMA FUNCTION
}

\author{
FENG QI AND CRISTINEL MORTICI
}

\begin{abstract}
In the paper, the authors establish three kinds of double inequalities for the trigamma function in terms of the exponential function to powers of the digamma function. These newly established inequalities extend some known results. The method in the paper utilizes some facts from the asymptotic theory and is a natural way to solve problems for approximating some quantities for large values of the variable.
\end{abstract}

\section{Motivations And main Results}

The classical Euler gamma function may be defined for $\Re(z)>0$ by

$$
\Gamma(z)=\int_{0}^{\infty} t^{z-1} e^{-t} \mathrm{~d} t .
$$

The logarithmic derivative of the gamma function $\Gamma(z)$ is denoted by

$$
\psi(z)=\frac{\mathrm{d}}{\mathrm{d} z}[\ln \Gamma(z)]=\frac{\Gamma^{\prime}(z)}{\Gamma(z)}
$$

and called the digamma function. The derivatives $\psi^{\prime}(z)$ and $\psi^{\prime \prime}(z)$ are called the trigamma and tetragamma functions respectively. As a whole, the functions $\psi^{(k)}(z)$ for $k \in\{0\} \cup \mathbb{N}$ are called the polygamma functions. These functions are widely used in theoretical and practical problems in all branches of mathematical science. Consequently, many mathematicians were preoccupied to establish new results about the gamma function, polygamma functions, and other related functions.

1.1. The first main result. In 2007, Alzer and Batir [3, Corollary] discovered that the double inequality

$$
\sqrt{2 \pi} x^{x} \exp \left[-x-\frac{1}{2} \psi(x+\alpha)\right]<\Gamma(x)<\sqrt{2 \pi} x^{x} \exp \left[-x-\frac{1}{2} \psi(x+\beta)\right]
$$

holds for $x>0$ if and only if $\alpha \geq \frac{1}{3}$ and $\beta \leq 0$. For information on generalizations of results in the paper [3], please refer to [12,32] and references cited therein. Motivated by the double inequality (1.1), Mortici [27] proposed the asymptotic formula

$$
\Gamma(x) \sim \sqrt{2 \pi} e^{-b}(x+b)^{x} \exp \left[-x-\frac{1}{2} \psi(x+c)\right], \quad x \rightarrow \infty
$$

and then determined the optimal values of parameters $b, c$ in such a way that this convergence is the fastest possible.

2010 Mathematics Subject Classification. Primary 33B15; Secondary 26A48, 26D15, 26D20.

Key words and phrases. inequality; digamma function; trigamma function; exponential function; asymptotic series.

This paper was typeset using $\mathcal{A M}_{\mathcal{S}}$-LATEX. 
In 2004, Batir [4, Theorem 2.1] presented the double inequality

$$
\begin{aligned}
\Gamma(c) \exp \left[\psi(x) e^{\psi(x)}-e^{\psi(x)}+1\right] & \leq \Gamma(x) \\
& \leq \Gamma(c) \exp \left\{\frac{6 e^{\gamma}}{\pi^{2}}\left[\psi(x) e^{\psi(x)}-e^{\psi(x)}+1\right]\right\}
\end{aligned}
$$

for every $x \geq c$, where $c=1.461 \ldots$ is the unique positive zero of the digamma function $\psi$ and $\gamma=0.577 \ldots$ is the Euler-Mascheroni constant. In 2010, Mortici [25] discovered the asymptotic formula

$$
\Gamma(x) \sim \frac{\sqrt{2 \pi}}{e} \exp \left[\psi(x) e^{\psi(x)}-e^{\psi(x)}+1\right], \quad x \rightarrow \infty .
$$

In 2011, the double inequality (1.2) was generalized by [11, Theorem 2] to a monotonicity property which reads that the function

$$
f_{s, t}(x)= \begin{cases}\frac{g_{s, t}(x)}{\left[g_{s, t}^{\prime}(x)-1\right] \exp \left[g_{s, t}^{\prime}(x)\right]+1}, & x \neq c \\ \frac{1}{g_{s, t}^{\prime \prime}(c)}, & x=c\end{cases}
$$

on $x \in(-\alpha, \infty)$ is decreasing for $|t-s|<1$ and increasing for $|t-s|>1$, where $s$ and $t$ are real numbers, $\alpha=\min \{s, t\}, c \in(-\alpha, \infty)$, and

$$
g_{s, t}(x)= \begin{cases}\frac{1}{t-s} \int_{c}^{x} \ln \left[\frac{\Gamma(u+t)}{\Gamma(u+s)} \frac{\Gamma(c+s)}{\Gamma(c+t)}\right] \mathrm{d} u, & s \neq t \\ \int_{c}^{x}[\psi(u+s)-\psi(c+s)] \mathrm{d} u, & s=t\end{cases}
$$

on $x \in(-\alpha, \infty)$.

In 2000, Elezović, Giordano, and Pečarić [8] found the single-sided inequality

$$
\psi^{\prime}(x)<e^{-\psi(x)}, \quad x>0
$$

This inequality is closely related to the monotonicity and convexity of the function

$$
\mathcal{Q}(x)=e^{\psi(x+1)}-x
$$

on $(-1, \infty)$. See also [13] and plenty of references therein. By the way, as a conjecture posed in [15, Remark 3.6], the complete monotonicity of the function $\mathcal{Q}(x)$ on $(0, \infty)$ still keeps open. An infinitely differentiable function $f$ is said to be completely monotonic on an interval $I$ if it satisfies $(-1)^{k} f^{(k)}(x) \geq 0$ on $I$ for all $k \geq 0$. This class of functions has applications in the approximation theory, asymptotic analysis, probability, integral transforms, and the like. See [7, Chapter 14], [22, Chapter XIII], [51, Chapter 1], and [52, Chapter IV]. For more information on the functions

$$
Q^{\prime}(x-1)=\psi^{\prime}(x) e^{\psi(x)}-1 \quad \text { and } \quad Q^{\prime \prime}(x-1)=\left\{\psi^{\prime \prime}(x)+\left[\psi^{\prime}(x)\right]^{2}\right\} e^{\psi(x)},
$$

please see the papers $[14,15,17,33,36,37,38,41,42,43,48,44,47,54,55]$, the expository and survey articles [34, 35, 45, 46], and a number of references cited therein. In 2011, Batir [5, Theorem 2.7] obtained the double inequality

$$
\left(x+a^{*}\right) e^{-2 \psi(x+1)}<\psi^{\prime}(x+1) \leq\left(x+b^{*}\right) e^{-2 \psi(x+1)}, \quad x>0,
$$


where the constants $a^{*}=\frac{1}{2}$ and $b^{*}=\frac{\pi^{2}}{6 e^{2 \gamma}}=0.518 \ldots$ are the best possible. In other words, Batir [5] proposed the approximation formula

$$
\psi^{\prime}(x+1) \sim(x+a) e^{-2 \psi(x+1)},
$$

where $a$ is a constant. A numerical computation shows that the approximation (1.5) gives a better result when choosing $a=a^{*}$, rather than the value $a=b^{*}$. This fact is somewhat expected as Batir obtained (1.4) as a result of the decreasing monotonicity of the function

$$
\theta(x)=\psi^{\prime}(x+1) e^{2 \psi(x+1)}-x
$$

on $(0, \infty)$, with $\theta(0)=\frac{\pi^{2}}{6 e^{2 \gamma}}$ and $\theta(\infty)=\frac{1}{2}$. Hence, the function $\theta(x)$ becomes gradually closer to $\theta(\infty)$, as $x$ approaches infinity. The decreasing monotonicity of the function $\theta(x)$ may be rewritten and extended as that the function

$$
\Theta(x)=\psi^{\prime}(x) e^{2 \psi(x)}-x+\frac{1}{2}
$$

is decreasing and positive, but not convex or concave, on $(0, \infty)$. In 2010 , among other things, Qi and Guo [43, Corollary 2] proved that the function

$$
f_{p, q}(x)=e^{p \psi(x+1)}-q x
$$

for $p \neq 0$ and $q \in \mathbb{R}$ is strictly convex with respect to $x \in(-1, \infty)$ if and only if $p \geq 1$ or $p<0$. Since

$$
\frac{\mathrm{d} e^{\alpha \psi(x)}}{\mathrm{d} x}=\alpha \psi^{\prime}(x) e^{\alpha \psi(x)} \quad \text { and } \quad \frac{\mathrm{d}^{2} e^{\alpha \psi(x)}}{\mathrm{d} x^{2}}=\alpha\left\{\psi^{\prime \prime}(x)+\alpha\left[\psi^{\prime}(x)\right]^{2}\right\} e^{\alpha \psi(x)},
$$

the above functions between (1.3) and (1.6) have something to do with the more general function

$$
f_{a, b, \alpha, \beta, \lambda}(x)=e^{a \psi(x+b)}+\alpha x^{2}+\beta x+\lambda
$$

and its monotonicity and convexity, even its complete monotonicity.

For obtaining accurate approximations of the type (1.5), the value $a=\frac{1}{2}$ should be used. However, approximations of the form

$$
\psi^{\prime}(x+1) \sim[x+a(x)] e^{-2 \psi(x+1)},
$$

with $a(x) \rightarrow \frac{1}{2}$ as $x$ tends to $\infty$, are much better. Furthermore, several experiments using some asymptotic expansions lead us to the claim that approximations of the type

$$
\psi^{\prime}(x+1) \sim[x+a(x)] \exp \left[-2 \psi(x+1)-\frac{1}{120 x^{4}}\right]
$$

are more accurate. Consequently, we obtain the following theorem.

Theorem 1.1. For $x \geq 3$, the double inequality

$$
\begin{aligned}
{[x+\alpha(x)] \exp \left[-2 \psi(x+1)-\frac{1}{120 x^{4}}\right] } & \leq \psi^{\prime}(x+1) \\
\leq & {[x+\beta(x)] \exp \left[-2 \psi(x+1)-\frac{1}{120 x^{4}}\right] }
\end{aligned}
$$

is valid, where

$$
\alpha(x)=\frac{1}{2}+\frac{1}{90 x^{3}}-\frac{1}{60 x^{4}} \quad \text { and } \quad \beta(x)=\frac{1}{2}+\frac{1}{90 x^{3}} .
$$


1.2. The second main result. In 2013, Guo and Qi [14, Lemma 2] proved that

$$
\psi^{\prime}(x)<e^{1 / x}-1, \quad x>0 .
$$

This inequality has been generalized to the complete monotonicity of the function

$$
e^{1 / x}-\psi^{\prime}(x)-1, \quad x>0
$$

and others. For detailed information, please refer to [16, 21, 39], [40, Theorem 3.1], [49, Theorem 1.1], [50, Theorem 1.1], and closely related references therein. In some of these references, it was obtained that

$$
\lim _{x \rightarrow \infty}\left[e^{1 / x}-\psi^{\prime}(x)\right]=1 .
$$

The approximation $\psi^{\prime}(x) \sim e^{1 / x}-1$ indeed gives a good result for the large value of $x$. Now we propose the improvement

$$
\psi^{\prime}(x) \sim e^{1 / x+\mu(x)}-1,
$$

where $\mu(x) \rightarrow 0$ and $\mu(x)=o(x)$ as $x \rightarrow \infty$. Our main result may be stated in details as the following theorem.

Theorem 1.2. For $x \geq 3$, we have

$$
e^{m(x)}-1<\psi^{\prime}(x)<e^{M(x)}-1,
$$

where

$$
m(x)=\frac{1}{x}-\frac{1}{24 x^{4}}+\frac{7}{360 x^{6}} \quad \text { and } \quad M(x)=m(x)+\frac{1}{90 x^{7}} .
$$

1.3. The third main result. In 2014, Yang, Chu, and Tao found [53, Theorem 1] that the constants $p=1$ and $q=2$ are the best possible real parameters such that the double inequality

$$
\theta(x, p)<\psi^{\prime}(x+1)<\theta(x, q)
$$

holds for $x>0$, where

$$
\theta(x, m)=\frac{e^{m /(x+1)}-e^{-m / x}}{2 m} .
$$

Because

$$
\psi^{\prime}(x+1)=\psi^{\prime}(x)-\frac{1}{x^{2}},
$$

the double inequality (1.11) may be reformulated as

$$
\frac{1}{x^{2}}+\theta(x, 1)<\psi^{\prime}(x)<\frac{1}{x^{2}}+\theta(x, 2), \quad x>0 .
$$

The complete monotonicity of the function (1.9) implies that

$$
e-\psi^{\prime}(1)>e^{1 /(x+1)}-\psi^{\prime}(x+1)>1, \quad x>0
$$

which may be rearranged as

$$
e^{1 /(x+1)}-e+\psi^{\prime}(1)<\psi^{\prime}(x+1)<e^{1 /(x+1)}-1<\frac{1}{2} \sinh \frac{2}{x}
$$

on $(0, \infty)$. When $t<1.6 \ldots$, the lower bound $e^{1 /(x+1)}-e+\psi^{\prime}(1)$ is better than the corresponding one in [53, Corollary 2]. The upper bound $e^{1 /(x+1)}-1$ in (1.14) and the upper bound $\theta(x, 2)$ in (1.11) can not be compared with each other. However, the upper bound $\frac{1}{x^{2}}+\theta(x, 2)$ in $(1.13)$ is better than the upper bound $e^{1 / x}-1$ in (1.8).

In this paper, we will improve the double inequality (1.11) as follows. 
Theorem 1.3. For $x \geq 1$, we have

$$
\theta(x, 1)+\frac{1}{24 x^{5}}-\frac{5}{48 x^{6}}<\psi^{\prime}(x+1)<\theta(x, 1)+\frac{1}{24 x^{5}}
$$

and

$$
\theta(x, 2)-\frac{1}{45 x^{7}}<\psi^{\prime}(x+1)<\theta(x, 2)-\frac{1}{45 x^{7}}+\frac{7}{90 x^{8}} .
$$

2. Proofs of Theorems 1.1 to 1.3

Now we start out to prove our three main results.

In 1997, Alzer [2, Theorem 8] proved that for $m, n \geq 1$ the functions

$$
F_{m}(x)=\ln \Gamma(x+1)-\left(x+\frac{1}{2}\right) \ln x+x-\frac{1}{2} \ln 2 \pi-\sum_{i=1}^{2 m} \frac{B_{2 i}}{2 i(2 i-1) x^{2 i-1}}
$$

and

$$
G_{n}(x)=-\ln \Gamma(x+1)+\left(x+\frac{1}{2}\right) \ln x-x+\frac{1}{2} \ln 2 \pi+\sum_{i=1}^{2 n-1} \frac{B_{2 i}}{2 i(2 i-1) x^{2 i-1}}
$$

are completely monotonic on $(0, \infty)$, where $B_{j}$ are the Bernoulli numbers given by the generating function

$$
\frac{t}{e^{t}-1}=\sum_{j=0}^{\infty} B_{j} \frac{t^{j}}{j !}=1-\frac{t}{2}+\sum_{j=0}^{\infty} B_{2 j} \frac{t^{2 j}}{(2 j) !} .
$$

See also [19, Theorem 2], [20, Theorem 2.1], and [31, Theorem 3.1]. From $F_{1}^{\prime}(x)<0$, $G_{2}^{\prime}(x)<0, F_{1}^{\prime \prime}(x)>0, F_{2}^{\prime \prime}(x)>0, G_{2}^{\prime \prime}(x)>0$, and $G_{3}^{\prime \prime}(x)>0$, it follows that

$$
\begin{gathered}
\ln x+\frac{1}{2 x}-\frac{1}{12 x^{2}}+\frac{1}{240 x^{4}}-\frac{1}{252 x^{6}}<\psi(x+1)<\ln x+\frac{1}{2 x}-\frac{1}{12 x^{2}}+\frac{1}{240 x^{4}}, \\
\psi^{\prime}(x+1)>\frac{1}{x}-\frac{1}{2 x^{2}}+\frac{1}{6 x^{3}}-\frac{1}{30 x^{5}} \\
\frac{1}{x}-\frac{1}{2 x^{2}}+\frac{1}{6 x^{3}}-\frac{1}{30 x^{5}}+\frac{1}{42 x^{7}}-\frac{1}{30 x^{9}}<\psi^{\prime}(x+1) \\
<\frac{1}{x}-\frac{1}{2 x^{2}}+\frac{1}{6 x^{3}}-\frac{1}{30 x^{5}}+\frac{1}{42 x^{7}},
\end{gathered}
$$

and, also by (1.12),

$$
\begin{aligned}
\frac{1}{x}+\frac{1}{2 x^{2}}+\frac{1}{6 x^{3}}-\frac{1}{30 x^{5}} & +\frac{1}{42 x^{7}}-\frac{1}{30 x^{9}}<\psi^{\prime}(x) \\
& <\frac{1}{x}+\frac{1}{2 x^{2}}+\frac{1}{6 x^{3}}-\frac{1}{30 x^{5}}+\frac{1}{42 x^{7}}-\frac{1}{30 x^{9}}+\frac{5}{66 x^{11}} .
\end{aligned}
$$

Proof of Theorem 1.1. By taking the logarithm, the double inequality (1.7) may be rearranged as

$$
F(x)=\ln [x+\alpha(x)]-2 \psi(x+1)-\ln \psi^{\prime}(x+1)-\frac{1}{120 x^{4}}<0
$$

and

$$
G(x)=-\ln [x+\beta(x)]+\ln \psi^{\prime}(x+1)+2 \psi(x+1)+\frac{1}{120 x^{4}}<0
$$


for $x \geq 3$. By virtue of inequalities (2.1) and (2.3), one may deduce that $F(x)<$ $F_{1}(x)$ and $G(x)<G_{1}(x)$, where

$$
\begin{aligned}
F_{1}(x)= & \ln [x+\alpha(x)]-2\left(\ln x+\frac{1}{2 x}-\frac{1}{12 x^{2}}+\frac{1}{240 x^{4}}-\frac{1}{252 x^{6}}\right) \\
& -\ln \left(\frac{1}{x}-\frac{1}{2 x^{2}}+\frac{1}{6 x^{3}}-\frac{1}{30 x^{5}}+\frac{1}{42 x^{7}}-\frac{1}{30 x^{9}}\right)-\frac{1}{120 x^{4}}
\end{aligned}
$$

and

$$
\begin{aligned}
G_{1}(x)= & -\ln [x+\beta(x)]+\ln \left(\frac{1}{x}-\frac{1}{2 x^{2}}+\frac{1}{6 x^{3}}-\frac{1}{30 x^{5}}+\frac{1}{42 x^{7}}\right) \\
& +2\left(\ln x+\frac{1}{2 x}-\frac{1}{12 x^{2}}+\frac{1}{240 x^{4}}\right)+\frac{1}{120 x^{4}} .
\end{aligned}
$$

Since

$$
F_{1}^{\prime}(x)=\frac{A(x-3)}{105 x^{7}\left(180 x^{5}+90 x^{4}+2 x-3\right) B(x-3)}
$$

and

where

$$
G_{1}^{\prime}(x)=\frac{C(x-3)}{15 x^{5}\left(90 x^{4}+45 x^{3}+1\right) D(x-3)},
$$

$$
\begin{aligned}
A(x)= & 84000 x^{13}+3753750 x^{12}+75109720 x^{11}+896904120 x^{10} \\
& +7160223140 x^{9}+40457327085 x^{8}+166700796732 x^{7}+507517074474 x^{6} \\
& +1141703970759 x^{5}+1873185114060 x^{4}+2175691772642 x^{3} \\
& +1690072075536 x^{2}+783944661553 x+162974708124, \\
B(x)= & 210 x^{8}+4935 x^{7}+50750 x^{6}+298305 x^{5}+1096193 x^{4} \\
& +2578821 x^{3}+3792857 x^{2}+3188649 x+1173161, \\
C(x)= & 23625 x^{9}+604200 x^{8}+6818475 x^{7}+44510545 x^{6} \\
& +184933335 x^{5}+506070905 x^{4}+909421185 x^{3} \\
& +1030441127 x^{2}+663679092 x+183168418, \\
D(x)= & 210 x^{6}+3675 x^{5}+26810 x^{4}+104370 x^{3}+228683 x^{2}+267393 x+130352
\end{aligned}
$$

are polynomials with positive coefficients, the functions $F_{1}(x)$ and $G_{1}(x)$ are strictly increasing on $[3, \infty)$. Furthermore, since

$$
\lim _{x \rightarrow \infty} F_{1}(x)=\lim _{x \rightarrow \infty} G_{1}(x)=0,
$$

it follows that $F_{1}(x)<0$ and $G_{1}(x)<0$ for $x \geq 3$. As a result, we have $F(x)<$ $F_{1}(x)<0$ and $G(x)<G_{1}(x)<0$. The proof of Theorem 1.2 is complete.

Proof of Theorem 1.2. The inequality (1.10) can be written as

$$
m(x)<\ln \left[1+\psi^{\prime}(x)\right]<M(x) .
$$

Considering the double inequality (2.4), since $m(x)-\ln \left[1+\psi^{\prime}(x)\right]<m_{1}(x)$ and $M(x)-\ln \left[1+\psi^{\prime}(x)\right]>M_{1}(x)$, one may see that it suffices to show that

$$
m_{1}(x) \triangleq m(x)-\ln \left(1+\frac{1}{x}+\frac{1}{2 x^{2}}+\frac{1}{6 x^{3}}-\frac{1}{30 x^{5}}+\frac{1}{42 x^{7}}-\frac{1}{30 x^{9}}\right)<0
$$


and

$$
M_{1}(x) \triangleq M(x)-\ln \left(1+\frac{1}{x}+\frac{1}{2 x^{2}}+\frac{1}{6 x^{3}}-\frac{1}{30 x^{5}}+\frac{1}{42 x^{7}}-\frac{1}{30 x^{9}}+\frac{5}{66 x^{11}}\right)>0 .
$$

A straightforward computation gives

$$
m_{1}^{\prime}(x)=\frac{E(x-3)}{60 x^{7} G(x-3)} \quad \text { and } \quad M_{1}^{\prime}(x)=-\frac{F(x-3)}{180 x^{8} H(x-3)},
$$

where

$$
\begin{aligned}
E(x)= & 980 x^{8}+22485 x^{7}+221130 x^{6}+1212855 x^{5}+4032099 x^{4} \\
& +8229303 x^{3}+9865371 x^{2}+6074127 x+1290163, \\
F(x)= & 66495 x^{10}+2146155 x^{9}+31007240 x^{8}+263913573 x^{7} \\
& +1464790565 x^{6}+5537745108 x^{5}+14437981040 x^{4} \\
& +25626153678 x^{3}+29624987873 x^{2}+20135221233 x+6106987838, \\
G(x)= & 210 x^{9}+5880 x^{8}+73185 x^{7}+531440 x^{6}+2481255 x^{5}+7724423 x^{4} \\
& +16033731 x^{3}+21398207 x^{2}+16660569 x+5765861, \\
H(x)= & 2310 x^{11}+78540 x^{10}+1213905 x^{9}+11258170 x^{8}+69614160 x^{7} \\
& +301344043 x^{6}+931827204 x^{5}+2058324400 x^{4}+3182887290 x^{3} \\
& +3281444518 x^{2}+2029943157 x+570820414
\end{aligned}
$$

are polynomials with positive coefficients. This means that $m_{1}^{\prime}(x)>0$ and $M_{1}^{\prime}(x)<$ 0 for $x \geq 3$, that is, the function $m_{1}(x)$ is strictly increasing and the function $M_{1}(x)$ is strictly decreasing on $[3, \infty)$, with the limits

$$
\lim _{x \rightarrow \infty} m_{1}(x)=\lim _{x \rightarrow \infty} M_{1}(x)=0 .
$$

Accordingly, one obtain that $m_{1}(x)<0$ and $M_{1}(x)>0$ on $[3, \infty)$. The proof of Theorem 1.2 is complete.

Proof of Theorem 1.3. The left hand side inequality in (1.15) can be written as

$$
c(x) \triangleq \frac{1}{2} e^{1 /(x+1)}-\frac{1}{2} e^{-1 / x}+\frac{1}{24 x^{5}}-\frac{5}{48 x^{6}}-\psi^{\prime}(x+1)<0 .
$$

Since

$$
e^{-1 / x}>\sum_{k=0}^{7} \frac{1}{k !}\left(-\frac{1}{x}\right)^{k}
$$

and the inequality (2.2) is valid, we have

$$
2 c(x)<e^{1 /(x+1)}-\frac{P(x)}{5040 x^{7}},
$$

where

$$
P(x)=5040 x^{7}+5040 x^{6}-2520 x^{5}+840 x^{4}+210 x^{3}-798 x^{2}+1057 x-1 .
$$

Hence, it is sufficient to prove that

$$
c_{1}(x)=\frac{1}{x+1}-\ln \frac{P(x)}{5040 x^{7}}<0 .
$$


A straightforward differentiation yields

$$
c_{1}^{\prime}(x)=\frac{7630 x^{2}+6329 x-7}{x(x+1)^{2} P(x)}=\frac{7630(x-1)^{2}+21589(x-1)+13952}{x(x+1)^{2} Q(x-1)},
$$

where

$$
\begin{aligned}
Q(x)= & 5040 x^{7}+40320 x^{6}+133560 x^{5}+240240 x^{4} \\
& +255570 x^{3}+161112 x^{2}+56371 x+8868 .
\end{aligned}
$$

This implies that the function $c_{1}(x)$ is strictly increasing on $[1, \infty)$. Furthermore, because of $\lim _{x \rightarrow \infty} c_{1}(x)=0$, it follows that $c_{1}(x)<0$ on $[1, \infty)$. The proof of the left hand side inequality in (1.15) is complete.

Similarly, we may verify other inequalities in (1.15) and (1.16). For the sake of saving the space and shortening the length of this paper, we do not repeat the processes. The proof of Theorem 1.3 is complete.

\section{REMARKS}

Finally we give several remarks on our three main results.

Remark 3.1. The right hand side in (1.7) is better than the right hand side in (1.4), but the left hand side in (1.7) is not better than the left hand side in (1.4). This is because the inequalities

$$
\left(x+a^{*}\right) e^{-2 \psi(x+1)}>[x+\alpha(x)] \exp \left[-2 \psi(x+1)-\frac{1}{120 x^{4}}\right]
$$

and

$$
[x+\beta(x)] \exp \left[-2 \psi(x+1)-\frac{1}{120 x^{4}}\right]<\left(x+b^{*}\right) e^{-2 \psi(x+1)},
$$

which are equivalent to

$$
u(x)=\ln \left(x+\frac{1}{2}+\frac{1}{90 x^{3}}-\frac{1}{60 x^{4}}\right)-\ln \left(x+\frac{1}{2}\right)-\frac{1}{120 x^{4}}<0
$$

and

$$
v(x)=\ln \left(x+\frac{1}{2}+\frac{1}{90 x^{3}}\right)-\frac{1}{120 x^{4}}-\ln \left(x+\frac{\pi^{2}}{6 e^{2 \gamma}}\right)<0,
$$

are valid for $x \geq 1$. The inequalities (3.1) and (3.2) may be verified as follows. A direct differentiation gives

$$
u^{\prime}(x)=\frac{540 x^{6}+60 x^{5}+180 x^{4}+90 x\left(x-\frac{1}{30}\right)^{2}+\frac{9}{10} x+2}{30 x^{5}(2 x+1) S\left(x-\frac{1}{10}\right)}>0
$$

for $x \geq \frac{1}{10}$ and

$$
v^{\prime}(x)=\frac{Q(x-1)}{30 x^{5}\left(90 x^{4}+45 x^{3}+1\right)\left(6 e^{2 \gamma} x+\pi^{2}\right)}>0
$$

for $x \geq 1$, where

$$
S(x)=180 x^{4}+162 x^{3}+\frac{189}{5} x^{2}+\frac{21}{50} x+\frac{226}{125}
$$

and

$$
\begin{aligned}
Q(x)= & 2700\left(\pi^{2}-3 e^{2 \gamma}\right) x^{8}+21600\left(\pi^{2}-3 e^{2 \gamma}\right) x^{7}+75600\left(\pi^{2}-3 e^{2 \gamma}\right) x^{6} \\
& +180\left(840 \pi^{2}-2521 e^{2 \gamma}\right) x^{5}+630\left(300 \pi^{2}-901 e^{2 \gamma}\right) x^{4}
\end{aligned}
$$




$$
\begin{aligned}
& +45\left(3361 \pi^{2}-10096 e^{2 \gamma}\right) x^{3}+45\left(1683 \pi^{2}-5044 e^{2 \gamma}\right) x^{2} \\
& +3\left(7245 \pi^{2}-21538 e^{2 \gamma}\right) x+2\left(1373 \pi^{2}-4002 e^{2 \gamma}\right)
\end{aligned}
$$

are polynomials with positive coefficients. This means that the functions $u(x)$ and $v(x)$ are strictly increasing on $[1, \infty)$. Further, from the limits

$$
\lim _{x \rightarrow \infty} u(x)=\lim _{x \rightarrow \infty} v(x)=0
$$

it follows that $u(x)<0$ on $(0, \infty)$ and $v(x)<0$ on $[1, \infty)$.

Remark 3.2 (The asymptotic series of $\left.\psi^{\prime}(x+1) e^{2 \psi(x+1)}\right)$. Whenever an approximation formula $f(x) \sim g(x)$ is considered in the sense that the ratio $\lim _{x \rightarrow \infty} \frac{f(x)}{g(x)}=1$ (sometimes $\lim _{x \rightarrow \infty}[f(x)-g(x)]=0$ ), there is a tendency to improve it by adding new terms, or an entire series, of the form

$$
f(x) \sim g(x)+\sum_{k=1}^{\infty} \frac{a_{k}}{x^{k}} .
$$

Such a series is called an asymptotic series and it plays a central role in the theory of approximation. Although such a series is often divergent, by truncating at the $m$-th term, it provides approximations of any desired accuracy $\frac{1}{x^{m+1}}$. In other words, the formula

$$
f(x) \sim g(x)+\sum_{k=1}^{m} \frac{a_{k}}{x^{k}}+O\left(\frac{1}{x^{m+1}}\right), \quad x \rightarrow \infty
$$

is valid for every integer $m \geq 1$.

It is well known [1] that the digamma and trigamma functions admit respectively the following asymptotic expansions:

$$
\psi(x+1) \sim \ln x+\frac{1}{2 x}-\sum_{k=2}^{\infty} \frac{B_{k}}{k x^{k}} \quad \text { and } \quad \psi^{\prime}(x+1) \sim-\frac{1}{x^{2}}+\sum_{k=1}^{\infty} \frac{B_{k-1}}{x^{k}} .
$$

The asymptotic expansions in (3.3) can be written explicitly as

$$
\psi(x+1) \sim \ln x+\frac{1}{2 x}-\frac{1}{12 x^{2}}+\frac{1}{240 x^{4}}-\frac{1}{252 x^{6}}+\frac{1}{240 x^{8}}-\frac{1}{132 x^{10}}+\cdots
$$

and

$$
\psi^{\prime}(x+1) \sim \frac{1}{x}-\frac{1}{2 x^{2}}+\frac{1}{6 x^{3}}-\frac{1}{30 x^{5}}+\frac{1}{42 x^{7}}-\frac{1}{30 x^{9}}+\frac{5}{66 x^{11}}-\cdots .
$$

In order to construct the asymptotic expansion of the function $\psi^{\prime}(x+1) e^{2 \psi(x+1)}$, we recall from the papers $[6,10]$ and the monographs $[9$, pp. 20-21] and [18, pp. 539-541] the following classical results from the theory of asymptotic series:

(1) if

as $x \rightarrow \infty$, then

$$
u(x) \sim \sum_{k=0}^{\infty} \frac{p_{k}}{x^{k}} \quad \text { and } \quad v(x) \sim \sum_{k=0}^{\infty} \frac{q_{k}}{x^{k}}
$$

$$
u(x) v(x) \sim \sum_{k=0}^{\infty} \frac{r_{k}}{x^{k}}
$$

where

$$
r_{k}=\sum_{i+j=k} p_{i} q_{j}
$$


(2) if

$$
f(x) \sim \sum_{k=0}^{\infty} \frac{a_{k}}{x^{k}}, \quad x \rightarrow \infty
$$

then

$$
\exp f(x) \sim \sum_{k=0}^{\infty} \frac{\alpha_{k}}{x^{k}}, \quad x \rightarrow \infty
$$

where $\alpha_{0}=\exp a_{0}$ and

$$
\alpha_{k}=\sum_{j=1}^{k} \frac{1}{j !} \sum_{i_{1}+\cdots+i_{j}=k} \prod_{\ell=1}^{j} a_{i_{\ell}}
$$

for $k \geq 1$.

Now the asymptotic series of the function $\psi^{\prime}(x+1) e^{2 \psi(x+1)}$ can be computed in two steps. Firstly, by virtue of the formula (3.5), we may transform the series (3.3) to obtain the series of $e^{2 \psi(x+1)}$. Secondly, with the help of the formula (3.4), we multiply the series of $e^{2 \psi(x+1)}$ and the second series in (3.3).

Because the general term (in terms of the Bernoulli numbers) of the series of the function $\psi^{\prime}(x+1) e^{2 \psi(x+1)}$ has an unattractive form, we just write down the first few terms as follows:

$$
\psi^{\prime}(x+1) e^{2 \psi(x+1)}=x+\frac{1}{2}+\frac{1}{90 x^{3}}-\frac{1}{60 x^{4}}+\frac{2}{567 x^{5}}+\frac{43}{2268 x^{6}}-O\left(\frac{1}{x^{7}}\right) .
$$

Till now we can see immediately that the functions $\alpha(x)$ and $\beta(x)$ in Theorem 1.1 are truncations of the series (3.6) at the first three or four terms.

When more terms of (3.6) are considered, the conclusion of Theorem 1.1 remains true and the estimates become more accurate.

Remark 3.3. We conjecture that the function $e^{M(x)}-\psi^{\prime}(x)-1$ is completely monotonic on $(0, \infty)$, but the function $\psi^{\prime}(x)-e^{m(x)}+1$ is not.

Remark 3.4. The reason why the double inequality (1.11) is of interest is because it provides much accurate estimates for the trigamma function $\psi^{\prime}(x)$ for large values of $x$, that is,

$$
\psi^{\prime}(x+1) \sim \theta(x, m), \quad x \rightarrow \infty .
$$

From (1.15) and (1.16), it is easily deduced that

$$
\psi^{\prime}(x+1)-\theta(x, 1)=O\left(\frac{1}{x^{5}}\right) \quad \text { and } \quad \psi^{\prime}(x+1)-\theta(x, 2)=O\left(\frac{1}{x^{7}}\right),
$$

then it is easy to see that the best approximation of the form (3.7) may be obtained by taking $m=2$.

Remark 3.5. Evidently, our inequalities (1.15) and (1.16) are much stronger than those in [53]. Our proofs for (1.15) and (1.16) are also much simpler than the original proof of [53, Theorem 1]. This shows that the natural approach to solve problems of approximating some quantities for large values of the variable is the theory of asymptotic series. This method or approach has been utilized and applied in the papers $[23,24,26,28,29,30]$, for examples.

Remark 3.6. In this paper, we essentially talk about the relations between inequalities, asymptotic approximations, and complete monotonicity. 
Acknowledgements. The first author was in part supported by the NNSF of China under Grant No. 11361038. The second author was partially supported by a Grant of the Romanian National Authority for Scientific Research, CNCSUEFISCDI, with the Project Number PN-II-ID-PCE-2011-3-0087.

\section{REFERENCES}

[1] M. Abramowitz and I. A. Stegun (Eds), Handbook of Mathematical Functions with Formulas, Graphs, and Mathematical Tables, National Bureau of Standards, Applied Mathematics Series 55, 10th printing, Dover Publications, New York and Washington, 1972.

[2] H. Alzer, On some inequalities for the gamma and psi functions, Math. Comp. 66 (1997), no. 217, 373-389; Available online at http://dx.doi.org/10.1090/S0025-5718-97-00807-7.

[3] H. Alzer and N. Batir, Monotonicity properties of the gamma function, Appl. Math. Lett. 20 (2007), no. 7, 778-781; Available online at http://dx.doi.org/10.1016/j.aml.2006.08.026.

[4] N. Batir, An interesting double inequality for Euler's gamma function, J. Inequal. Pure Appl. Math. 5 (2004), no. 4, Art. 97; Available online at http://www.emis.de/journals/ JIPAM/article452.html.

[5] N. Batir, Sharp bounds for the psi function and harmonic numbers, Math. Inequal. Appl. 14 (2011), no. 4, 917-925; Available online at http://dx.doi.org/10.7153/mia-14-77.

[6] C.-P. Chen, N. Elezović, and L. Vukšić, Asymptotic formulae associated with the Wallis power function and digamma function, J. Classical Anal. 2 (2013), no. 2, 151-166; Available online at http://dx.doi.org/10.7153/jca-02-13.

[7] W. Cheney and W. Light, A Course in Approximation Theory, Reprint of the 2000 original, Graduate Studies in Mathematics, Volume 101, American Mathematical Society, Providence, RI, 2009.

[8] N. Elezović, C. Giordano, and J. Pečarić, The best bounds in Gautschi's inequality, Math. Inequal. Appl. 3 (2000), 239-252; Available online at http://dx.doi.org/10.7153/mia-03-26.

[9] A. Erdélyi, Asymptotic Expansions, Dover Publications, Inc., New York, 1956.

[10] H. W. Gould, Coefficient identities for powers of Taylor and Dirichlet series, Amer. Math. Monthly 81 (1974), no. 1, 3-14; Available online at http://www.jstor.org/stable/2318904.

[11] B.-N. Guo and F. Qi, A class of completely monotonic functions involving divided differences of the psi and tri-gamma functions and some applications, J. Korean Math. Soc. 48 (2011), no. 3, 655-667; Available online at http://dx.doi.org/10.4134/JKMS.2011.48.3.655.

[12] B.-N. Guo and F. Qi, A class of completely monotonic functions involving the gamma and polygamma functions, Cogent Math. 1 (2014), 1:982896, 8 pages; Available online at http: //dx.doi.org/10.1080/23311835.2014.982896.

[13] B.-N. Guo and F. Qi, An alternative proof of Elezović-Giordano-Pečarić's theorem, Math. Inequal. Appl. 14 (2011), no. 1, 73-78; Available online at http://dx.doi.org/10.7153/ mia-14-06.

[14] B.-N. Guo and F. Qi, Refinements of lower bounds for polygamma functions, Proc. Amer. Math. Soc. 141 (2013), no. 3, 1007-1015; Available online at http://dx.doi.org/10.1090/ S0002-9939-2012-11387-5.

[15] B.-N. Guo and F. Qi, Sharp inequalities for the psi function and harmonic numbers, Analysis (Berlin) 34 (2014), no. 2, 201-208; Available online at http://dx.doi.org/10.1515/ anly-2014-0001.

[16] B.-N. Guo and F. Qi, Some properties of the psi and polygamma functions, Hacet. J. Math. Stat. 39 (2010), no. 2, 219-231.

[17] B.-N. Guo, F. Qi, and H. M. Srivastava, Some uniqueness results for the non-trivially complete monotonicity of a class of functions involving the polygamma and related functions, Integral Transforms Spec. Funct. 21 (2010), no. 11, 849-858; Available online at http://dx.doi.org/10.1080/10652461003748112.

[18] K. Knopp, Theory and Application of Infinite Series, Second English Edition, translated from the Fourth German Edition by Miss R. C. H. Young, Blackie \& Son Limited, GlascowBombay-Toronto, 1951.

[19] S. Koumandos, Remarks on some completely monotonic functions, J. Math. Anal. Appl. 324 (2006), no. 2, 1458-1461; Available online at http://dx.doi.org/10.1016/j.jmaa.2005.12. 017. 
[20] S. Koumandos and H. L. Pedersen, Completely monotonic functions of positive order and asymptotic expansions of the logarithm of Barnes double gamma function and Euler's gamma function, J. Math. Anal. Appl. 355 (2009), no. 1, 33-40; Available online at http://dx.doi. org/10.1016/j.jmaa.2009.01.042.

[21] W.-H. Li, F. Qi, and B.-N. Guo, On proofs for monotonicity of a function involving the psi and exponential functions, Analysis (Munich) 33 (2013), no. 1, 45-50; Available online at http://dx.doi.org/10.1524/anly.2013.1175.

[22] D. S. Mitrinović, J. E. Pečarić, and A. M. Fink, Classical and New Inequalities in Analysis, Kluwer Academic Publishers, Dordrecht-Boston-London, 1993.

[23] C. Mortici, A continued fraction approximation of the gamma function, J. Math. Anal. Appl. 402 (2013), no. 2, 405-410; Available online at http://dx.doi.org/10.1016/j.jmaa.2012. 11.023.

[24] C. Mortici, A subtly analysis of Wilker inequality, Appl. Math. Comp. 231 (2014), 516-520; Available online at http://dx.doi.org/10.1016/j.amc.2014.01.017.

[25] C. Mortici, Estimating gamma function by digamma function, Math. Comput. Modelling 52 (2010), no. 5-6, 942-946; Available online at http://dx.doi.org/10.1016/j.mcm.2010.05. 030.

[26] C. Mortici, New approximation formulas for evaluating the ratio of gamma functions, Math. Comp. Modelling 52 (2010), no. 1-2, 425-433; Available online at http://dx.doi.org/10. 1016/j.mcm.2010.03.013.

[27] C. Mortici, New approximations of the gamma function in terms of the digamma function, Appl. Math. Lett. 23 (2010), no. 3, 97-100; Available online at http://dx.doi.org/10.1016/ j.aml.2009.08.012.

[28] C. Mortici, New improvements of the Stirling formula, Appl. Math. Comput. 217 (2010), no. 2, 699-704; Available online at http://dx.doi.org/10.1016/j.amc.2010.06.007.

[29] C. Mortici, Ramanujan formula for the generalized Stirling approximation, Appl. Math. Comp. 217 (2010), no. 6, 2579-2585; Available online at http://dx.doi.org/10.1016/j. amc. 2010.07.070.

[30] C. Mortici, The natural approach of Wilker-Cusa-Huygens inequalities, Math. Inequal. Appl. 14 (2011), no. 3, 535-541; Available online at http://dx.doi.org/10.7153/mia-14-46.

[31] C. Mortici, Very accurate estimates of the polygamma functions, Asympt. Anal. 68 (2010), no. 3, 125-134; Available online at http://dx.doi.org/10.3233/ASY-2010-0983.

[32] F. Qi, A completely monotonic function involving the gamma and tri-gamma functions, available online at http://arxiv.org/abs/1307.5407.

[33] F. Qi, A completely monotonic function related to the q-trigamma function, Politehn. Univ. Bucharest Sci. Bull. Ser. A Appl. Math. Phys. 76 (2014), no. 1, 107-114.

[34] F. Qi, Bounds for the ratio of two gamma functions, J. Inequal. Appl. 2010 (2010), Article ID 493058, 84 pages; Available online at http://dx.doi.org/10.1155/2010/493058.

[35] F. Qi, Bounds for the ratio of two gamma functions: from Gautschi's and Kershaw's inequalities to complete monotonicity, Turkish J. Anal. Number Theory 2 (2014), no. 5, 152-164; Available online at http://dx.doi.org/10.12691/tjant-2-5-1.

[36] F. Qi, Complete monotonicity of functions involving the q-trigamma and q-tetragamma functions, Rev. R. Acad. Cienc. Exactas F ís. Nat. Ser. A Mat. RACSAM 109 (2015), no. 2, in press; Available online at http://dx.doi.org/10.1007/s13398-014-0193-3.

[37] F. Qi, Completely monotonic degree of a function involving the tri- and tetra-gamma functions, available online at http://arxiv.org/abs/1301.0154.

[38] F. Qi, Integral representations and complete monotonicity related to the remainder of Burnside's formula for the gamma function, J. Comput. Appl. Math. 268 (2014), 155-167; Available online at http://dx.doi.org/10.1016/j.cam.2014.03.004.

[39] F. Qi, Properties of modified Bessel functions and completely monotonic degrees of differences between exponential and trigamma functions, Math. Inequal. Appl. 18 (2015), no. 2, 493-518; Available online at http://dx.doi.org/10.7153/mia-18-37.

[40] F. Qi and C. Berg, Complete monotonicity of a difference between the exponential and trigamma functions and properties related to a modified Bessel function, Mediterr. J. Math. 10 (2013), no. 4, 1685-1696; Available online at http://dx.doi.org/10.1007/ s00009-013-0272-2. 
[41] F. Qi, P. Cerone, and S. S. Dragomir, Complete monotonicity of a function involving the divided difference of psi functions, Bull. Aust. Math. Soc. 88 (2013), no. 2, 309-319; Available online at http://dx.doi.org/10.1017/S0004972712001025.

[42] F. Qi and B.-N. Guo, Completely monotonic functions involving divided differences of the di- and tri-gamma functions and some applications, Commun. Pure Appl. Anal. 8 (2009), no. 6, 1975-1989; Available online at http://dx.doi.org/10.3934/cpaa.2009.8.1975.

[43] F. Qi and B.-N. Guo, Necessary and sufficient conditions for functions involving the triand tetra-gamma functions to be completely monotonic, Adv. Appl. Math. 44 (2010), no. 1, 71-83; Available online at http://dx.doi.org/10.1016/j.aam.2009.03.003.

[44] F. Qi, B.-N. Guo, and C.-P. Chen, The best bounds in Gautschi-Kershaw inequalities, Math. Inequal. Appl. 9 (2006), no. 3, 427-436; Available online at http://dx.doi.org/10.7153/ mia-09-41.

[45] F. Qi and Q.-M. Luo, Bounds for the ratio of two gamma functions-From Wendel's and related inequalities to logarithmically completely monotonic functions, Banach J. Math. Anal. 6 (2012), no. 2, 132-158.

[46] F. Qi and Q.-M. Luo, Bounds for the ratio of two gamma functions: from Wendel's asymptotic relation to Elezović-Giordano-Pečarić's theorem, J. Inequal. Appl. 2013, 2013:542, 20 pages; Available online at http://dx.doi.org/10.1186/1029-242X-2013-542.

[47] F. Qi and Q.-M. Luo, Complete monotonicity of a function involving the gamma function and applications, Period. Math. Hungar. 69 (2014), no. 2, 159-169; Available online at http: //dx.doi.org/10.1007/s10998-014-0056-x.

[48] F. Qi, Q.-M. Luo, and B.-N. Guo, Complete monotonicity of a function involving the divided difference of digamma functions, Sci. China Math. 56 (2013), no. 11, 2315-2325; Available online at http://dx.doi.org/10.1007/s11425-012-4562-0.

[49] F. Qi and S.-H. Wang, Complete monotonicity, completely monotonic degree, integral representations, and an inequality related to the exponential, trigamma, and modified Bessel functions, Glob. J. Math. Anal. 2 (2014), no. 3, 91-97; Available online at http://dx.doi. org/10.14419/gjma.v2i3.2919.

[50] F. Qi and X.-J. Zhang, Complete monotonicity of a difference between the exponential and trigamma functions, J. Korea Soc. Math. Educ. Ser. B Pure Appl. Math. 21 (2014), no. 2, 141-145; Available online at http://dx.doi.org/10.7468/jksmeb.2014.21.2.141.

[51] R. L. Schilling, R. Song, and Z. Vondraček, Bernstein Functions-Theory and Applications, 2nd ed., de Gruyter Studies in Mathematics 37, Walter de Gruyter, Berlin, Germany, 2012; Available online at http://dx.doi.org/10.1515/9783110269338.

[52] D. V. Widder, The Laplace Transform, Princeton University Press, Princeton, 1946.

[53] Z.-H. Yang, Y.-M. Chu, and X.-J. Tao, A double inequality for the trigamma function and its applications, Abstr. Appl. Anal. 2014 (2014), Article ID 702718, 9 pages; Available online at http://dx.doi.org/10.1155/2014/702718.

[54] J.-L. Zhao, B.-N. Guo, and F. Qi, A completely monotonic function involving the tri- and tetra-gamma functions, Math. Slovaca 63 (2013), no. 3, 469-478; Available online at http: //dx.doi.org/10.2478/s12175-013-0109-2.

[55] J.-L. Zhao, B.-N. Guo, and F. Qi, Complete monotonicity of two functions involving the tri- and tetra-gamma functions, Period. Math. Hungar. 65 (2012), no. 1, 147-155; Available online at http://dx.doi.org/10.1007/s10998-012-9562-x.

(Qi) Institute of Mathematics, Henan Polytechnic University, Jiaozuo City, Henan Province, 454010, China; College of Mathematics, Inner Mongolia University for Nationalities, Tongliao City, Inner Mongolia Autonomous Region, 028043, China; Department of Mathematics, College of Science, Tianjin Polytechnic University, Tianjin City, 300387, CHINA

E-mail address: qifeng618@gmail.com, qifeng618@hotmail.com, qifeng618@qq.com

$U R L:$ http://qifeng618. wordpress . com

(Mortici) Department of Mathematics, Valahia University of Târgoviş̧e, Bd. Uniri 18, 130082 Târgovişte, Romania; Academy of Romanian Scientists, Splaiul Independen TुEI 54, 050094 Bucharest, ROMANiA

E-mail address: cristinel.mortici@hotmail.com, cmortici@valahia.ro

$U R L:$ http://www.cristinelmortici.ro 